\title{
Support Vector Machines for Knowledge Discovery
}

\author{
Shinsuke Sugaya ${ }^{1}$, Einoshin Suzuki $^{1}$, and Shusaku Tsumoto ${ }^{2}$ \\ 1 Division of Electrical and Computer Engineering, Faculty of Engineering, \\ Yokohama National University, \\ $\{$ shinsuke, suzuki\}@slab.dnj.ynu.ac.jp \\ 2 Department of Medical Informatics, Shimane Medical University, \\ School of Medicine, \\ tsumoto@computer.org
}

\begin{abstract}
In this paper, we apply support vector machine (SVM) to knowledge discovery (KD) and confirm its effectiveness with a benchmark data set. SVM has been successfully applied to problems in various domains. However, its effectiveness as a KD method is unknown. We propose SVM for KD, which deals with a classification problem with a binary class, by rescaling each attribute based on z-scores. SVM for $\mathrm{KD}$ can sort attributes with respect to their effectiveness in discriminating classes. Moreover, SVM for KD can discover crucial examples for discrimination. We settled six discovery tasks with the meningoencephalitis data set, which is a benchmark data set in KD. A domain expert ranked the discovery outcomes of SVM for KD from one to five with respect to several criteria. Selected attributes in six tasks are all valid and useful: their average scores are 3.8-4.0. Discovering order of attributes about usefulness represents a challenging problem. However, concerning this problem, our method achieved a score of more than or equal to 4.0 in three tasks. Besides, crucial examples for discrimination and typical examples for each class agree with medical knowledge. These promising results demonstrate the effectiveness of our approach.
\end{abstract}

\section{Introduction}

Support vector machine (SVM) $[1,10]$ is an effective method for a classification problem with a binary class. Although SVM has been successful in various domains $[2,5,6]$, its effectiveness as a knowledge discovery (KD) method is unknown. Since SVM has achieved high accuracy in various domains, we considered that applying SVM to KD and extracting information from its accurate model would likely to produce useful knowledge.

However, if SVM is directly applied to a date set, it is difficult to determine effective attributes for discrimination since an OSH depends on scales for attribute values. In order to circumvent this problem, we propose SVM for KD that adjusts these scales to an approximately equivalent length based on z-scores [3]. SVM for KD obtains effective attributes for discrimination, crucial examples for discrimination and typical examples for each class. 
We have applied SVM for KD to meningoencephalitis data set [8], which is a common problem in KD created by one of the authors, Tsumoto (a medical doctor). We performed experiments on six problems by selecting six attributes as a class. Tsumoto judged quantitatively that all the results with respect to attributes are useful. He also confirmed that results concerning crucial examples and typical examples agree with medical knowledge.

\section{SuperKDD: Discovery Challenge Contest}

The goal of SuperKDD(Supervised KDD) [8] is to compare KD methods under the supervision of a domain expert (Tsumoto) using a common medical data set such as meningoencephalitis data set. The results of SuperKDD enable us to characterize KD methods more concretely, and show the importance of interaction between KD researchers and domain experts.

The common data set collects the data of patients who suffered from meningitis and were admitted to the department of emergency and neurology in several hospitals. Tsumoto worked as a domain expert for these hospitals and collected those data from the past patient records (1979 to 1989) and the cases in which he made a diagnosis (1990 to 1993).

The database consists of 140 cases and all the data are described by 38 attributes, including present and past history, laboratory examinations, final diagnosis, therapy, clinical courses and final status after the therapy.

\section{$3 \quad$ SVM for Knowledge Discovery}

\subsection{Support Vector Machines}

Support vector machines $[1,10]$ are a new learning method introduced by V.Vapnik and AT\&T Bell Laboratories. SVM is applied to a classification problem with a binary class, and finds a classification model that discriminates each vector belonging to two classes. In SVM, a vector that is the closest to a hyperplane is called a support vector (SV). The classification model, the optimal separating hyperplane $(\mathrm{OSH})$, is given by maximizing the distance between the hyperplane and a SV. SVM has been applied to various problems and achieved higher accuracy than the conventional methods $[2,6]$.

\subsection{Description of SVM for KD}

Information obtained from SVM is the OSH, SVs, non-SVs and misclassified examples. This paper considers exploiting a normal vector of the OSH, SVs and non-SVs for KD.

We first explain how to use the normal vector of the OSH. Consider an attribute of which coefficient in the normal vector has a large absolute value. Figure 1(a) illustrates the projection of examples on the axis of this attribute in the example space. From figure 1(a), we see that examples can be discriminated by this 
attribute. We can conclude that, an absolute value of the coefficient represents discrimination power of its attribute. Here, in figure 1(b), readers may consider that a classification model which is represented by a dotted line is produced and an attribute of which coefficient has a small absolute value influences the discrimination. However, this case never happens since SVM produces a classification model that is represented by a straight line, which has a larger margin than the dotted line.

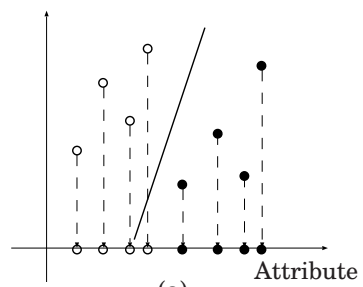

(a)

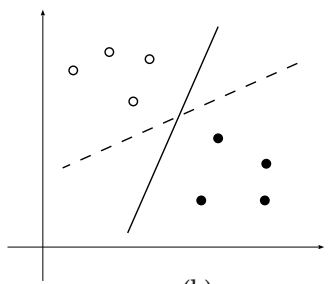

(b)

Fig. 1. (a) A projection of the examples on the axis of an attribute of which coefficient in the normal vector has a large absolute value. (b) A counter example for a discriminative attribute of which coefficient has a small absolute value. The straight line, not the dotted line, is produced as the OSH.

The above discussions hold true for attributes of which scales are approximately equivalent. An absolute value of a coefficient in the normal vector also depends on the scale of the attribute. In order to understand this, consider the example in figure 2. The left-hand side (a) and the right-hand side (b) represent expressing an attribute "HEIGHT" in inch or in centimeter respectively. We see that, although these represent the same attribute, the line in the right-hand side is flatter due to the smaller scale for "HEIGHT". Hence, if scales of each attribute are totally different, it is difficult to determine effective attributes for discrimination.

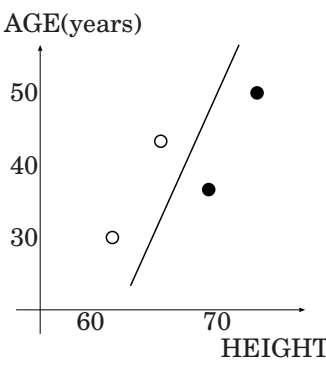

(a) (inch)

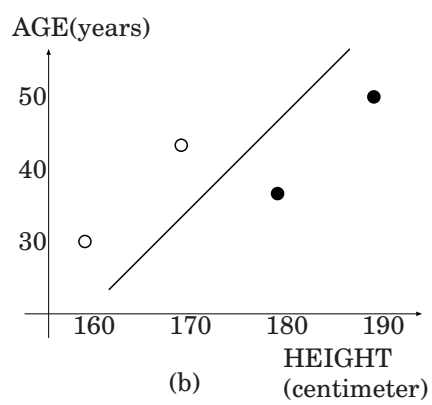

(b)

Fig. 2. Effect of expressing an attribute "HEIGHT" in centimeter (a) or in inch (b) respectively. Line (b) is flatter due to its smaller scale for values. 
In order to circumvent this problem, we propose to normalize each attribute by z-scores [3]. Z-scores is a method for obtaining a relative position of the data with respect to an attribute. A value of $\mathrm{z}$-scores $z_{i}$ represents the number of standard deviation $s$ between an attribute value $x_{i}$ and an average value $x_{m}$. Attribute values are transformed by

$$
z_{i}=\frac{x_{i}-x_{m}}{s}
$$

Effective attributes for discrimination are determined by this transformation, which corresponds to adjusting attributes to approximately equivalent scales.

SVM for KD obtains $n$ most-effective attributes for discrimination by calculating coefficients of the normal vector of the OSH and sorting them with respect to their absolute values.

Next, we explain how to use SVs and non-SVs. A SV, which is the closest example to the $\mathrm{OSH}$, is an example that determines each class. Hence, a SV corresponds to a crucial example for discrimination. On the other hand, since a non-SV is farther from the OSH than a SV, it can be considered as a typical example for its class. SVM for KD outputs SVs as crucial examples for discrimination and non-SVs as typical examples for each class.

\section{Application to SuperKDD}

\subsection{Conditions}

We applied SVM for KD to meningoencephalitis data set [8]. We settled six tasks by selecting Diag2 (grouped attribute of diagnosis), EEG_WAVE (electroencephalography), CT_FIND (CT finding), CULT_FIND (whether bacteria or virus is specified or not), COURSE (grouped attribute of clinical course at discharge) and RISK (grouped attribute of risk factor) as a class. Since SVM can be applied to only continuous attributes, nominal attributes with more than two values were ignored in the experiments. Here, attributes concerning therapy and courses, such as COURSE, can be measured only after diagnosis. They were ignored in four tasks with respect to Diag2, EEG_WAVE, CT_FIND and CULT_FIND since they are not available in determining these classes. Moreover, RISK was removed for data analysis because it was selected as a decision attribute.

After the experiments shown in [9], we generalized several attributes, which have too many values and seem to lose essential information for data analysis. Also, categorical attributes were tranformed to numerical attributes for SVM. We applied SVM after all preprocessing procedures. So, the results obtained here cannot be compared directly with those shown in the former paper, where such preprocessing procedures were not applied.

\subsection{Effective Attribute for Discrimination}

We first justify our rescaling strategy for attributes by comparing results with and without z-scores transformations. Ten hightest-ranking attributes for discri- 
mination with respect to class Diag2 with and without z-scores transformations are $\{$ Cell_Poly, CSF_CELL, CRP, STIFF, KERNIG, Cell_Mono, CT_FIND, CSF_GLU, COLD, WBC $\}$ and $\{\mathrm{CT}$ _FIND, FOCAL, STIFF, CRP, KERNIG, SEIZURE, BT, GCS, COLD, LOC $\}$ respectively. As described in section 3.2, attributes each of which scale is small, such as Cell_Poly and CSF_CELL, are not obtained without z-scores transformations. However, domain knowledge describes that these attributes are effective in this discrimination task. These results demonstrate validness of our approach.

Table 1. Average scores of ten hightest-ranking attributes concerning three criteria in six tasks with different classes, where "unexpect." represents unexpectedness.

\begin{tabular}{|c|c|c|c|c|c|c|}
\hline \multirow[b]{2}{*}{ Class } & \multicolumn{3}{|c|}{ Selection of Attributes } & \multicolumn{3}{|c|}{ Order of Attributes } \\
\hline & Validness & Unexpect. & Usefulness & Validness & Unexpect. & Usefulness \\
\hline Diag2 & 4.8 & 1.0 & 4.8 & 4.0 & 1.4 & 4.2 \\
\hline EEG_WAVE & 4.7 & 1.2 & 4.1 & 3.0 & 2.7 & 3.2 \\
\hline CT_FIND & 4.3 & 1.7 & 4.1 & 3.3 & 1.4 & 3.2 \\
\hline CULT_FIND & 4.4 & 1.8 & 4.1 & 3.3 & 2.6 & 3.6 \\
\hline COURSE & 5.0 & 1.0 & 5.0 & 2.9 & 2.3 & 4.0 \\
\hline RISK & 3.8 & 2.5 & 4.0 & 2.6 & 2.4 & 4.0 \\
\hline
\end{tabular}

Next, the results obtained by applying SVM for KD to six tasks are summarized in Table 1. Tsumoto, a domain expert, ranked, from one to five, selected attributes and order of attributes concerning ten hightest-ranking attributes with respect to validness, unexpectedness and usefulness. Here, validness indicates that discovered results agree with the medical context, and unexpectedness represents that discovered results can be partially explained by the medical context but are not accepted as common sense. Usefulness indicates that discovered results are useful in medical context. According to Tsumoto, it is relatively difficult to select ten relevant attributes in this problem.

For selection of attributes, we see that the results are satisfactory. All the average scores concerning usefulness are more than or equal to 4.0 and the scores concerning validness in five tasks are more than or equal to 4.3. Scores are rather low for unexpectedness, however, the meaning of this index is different from the other two. From the left-hand side of table 1, we can conclude that our method always discovered useful attributes for discrimination in these six tasks, and the selections were rather conservative than exploratory.

Determining order of attributes represents a more difficult problem than simply selecting attributes. From the table, our method is still effective for this problem. For validness, scores are more than or equal to 3.0 except for the task for COURSE and RISK. In terms of usefulness, our SVM for KD always achieved a score of more than or equal to 3.2. Especially, in three out of six tasks, scores are more than or equal to 4.0. Scores about unexpectedness are higher than 
in attribute-selection problem. However, our method can be still classified as conservative.

The results may be summarized as follows: pieces of knowledge obtained by SVM for KD are all useful with respect to selected attributes. Moreover, they are promising for a more difficult problem: determining order of attributes. For both problems, SVM for KD has turned out to be conservative: obtained results are expected. However, this tendency would be important in domains such as medical diagnosis.

\subsection{Classification of Examples}

Table 2. Performance for selecting crucial examples for discrimination and typical examples for each class. Each ratio indicates how many examples agree with medical context.

\begin{tabular}{|c|c|c|c|c|c|}
\hline Diag2 & G_W & T_FII & $\overline{L T}$ TF & OUR & RIS \\
\hline $90 \%$ & $100 \%$ & $100 \%$ & $79 \%$ & $89 \%$ & $100^{\circ}$ \\
\hline Non-SV $100 \%$ & $100 \%$ & $100 \%$ & $95 \%$ & $100 \%$ & 99 \\
\hline
\end{tabular}

As described in section 3, SVs are considered as crucial examples for discrimination. Remarkably, from table 2, Tsumoto judged that all the examples obtained in three tasks correspond exactly to crucial examples for discrimination in medical context. Moreover, 79\%-90\% of examples concerning the remaining tasks agree with medical context. On the other hand, non-SVs are considered typical examples for each class. Remarkly, 95\%-100\% of examples in six tasks also agree with medical context. These results clearly demonstrate the effectiveness of our approach for this problem.

We also investigated misclassified examples. There were relatively many misclassified examples in two tasks: CULT_FIND and COURSE. Tsumoto explained that these tasks are difficult for a linear discrimination-model such as our SVM, and these misclassifications are reasonable. Since our method can discover effective attributes and crucial examples in discrimination as demonstrated in section 4.2 , we do not consider that these misclassified examples should be decreased in number. However, outliers and exceptions are gaining increasing attention in $\mathrm{KD}$ community [4,7]. We regard these examples as interesting, and are currently planning to exploit them for KD.

\section{Conclusions}

This paper has explored the capability of support vector machine $[1,10]$ in knowledge discovery. Three types of knowledge in discrimination, effective attributes, crucial examples and typical examples are discovered in our approach. We have proposed attribute-rescaling based on z-scores [3] for this purpose. 
Meningoencephalitis data set [8], which is used in a contest of knowledge discovery methods, was chosen to demonstrate the effectiveness of our approach. One of the authors, Tsumoto, who is also a medical doctor, quantitatively evaluated the results. From the results, we can safely conclude that our method discovers useful attributes for discrimination, crucial examples and typical examples that are equivalent to medical knowledge. Ongoing work is focused on the exploitation of misclassified examples in knowledge discovery, and investigation of non-linear models generated by kernel methods $[1,10]$ with this data set.

\section{Acknowledgement}

This work was partially supported by the grant-in-aid for scientific research on priority area "Discovery Science" from the Japanese Ministry of Education, Science, Sports and Culture (11130207).

\section{References}

1. C.Cortes and V.Vapnik: "Support Vector Network", Machine Learning, Vol.20, No. 3, pp.1-25, 1995.

2. T.Joachims: "Text Categorization with Support Vector Machines: Learning with Many Relevant Features", Proc. Tenth European Conf. Machine Learning (ECML), pp.137-142, 1998.

3. L.Kaufman and P.Rousseeuw: Finding Groups in Data, John Wiley \& Sons, 1990.

4. E.M.Knorr and R.T.Ng: "Algorithms for Mining Distance-Based Outliers in Large Datasets", Proc. 24th Ann. Int'l Conf. Very Large Data Bases (VLDB), pp.392403, 1998.

5. E.Osuna, R.Freund and F.Girosi: "Training Support Vector Machines: an Application to Face Detection", Proc. Computer Vision and Pattern Recognition, pp.130136, 1997.

6. M.Pontil and A.Verri: "Support Vector Machines for 3D Object Recognition", IEEE Trans. Pattern Analysis and Machine Intelligence, Vol.20, No.6, pp.637-646, 1998.

7. E.Suzuki: "Autonomous Discovery of Reliable Exception Rules", Proc. Third Int'l Conf. Knowledge Discovery and Data Mining (KDD), pp.259-262, 1997.

8. S.Tsumoto, et al.: "Comparison of Data Mining Methods using Common Medical Datasets", ISM Symp.: Data Mining and Knowledge Discovery in Data Science, pp. 63-72, 1999.

9. S.Tsumoto: "Knowledge Discovery in Clinical Databases: an Experiment with Rule Induction and Statistics", Proc. Eleventh Int'l Symp. Methodologies for Intelligent Systems (ISMIS), pp.349-357, LNAI 1609, Springer-Verlag, 1999.

10. V.Vapnik: The Nature of Statistical Learning Theory, Springer-Verlag, New York, 1995. 